University of Wollongong

Research Online

Faculty of Social Sciences - Papers (Archive) Faculty of Arts, Social Sciences \& Humanities

2019

Time Effects on Resting EEG in Children With/Without AD/HD

Dawei Zhang

University of Wollongong, dwz847@uowmail.edu.au

Stuart J. Johnstone

University of Wollongong, sjohnsto@uow.edu.au

Hui Li

Peking University

Robert J. Barry

University of Wollongong, rbarry@uow.edu.au

Adam R. Clarke

University of Wollongong, aclarke@uow.edu.au

See next page for additional authors

Follow this and additional works at: https://ro.uow.edu.au/sspapers

Part of the Education Commons, and the Social and Behavioral Sciences Commons

Research Online is the open access institutional repository for the University of Wollongong. For further information contact the UOW Library: research-pubs@uow.edu.au 


\title{
Time Effects on Resting EEG in Children With/Without AD/HD
}

\author{
Abstract \\ In this study we extend on behavioural evidence to examine the effect of time on EEG measures related to \\ arousal and emotion/motivation in children with/without AD/HD. Thirty children with AD/HD and 30 age- \\ and sex-matched controls participated. EEG was recorded during an eyes-closed resting condition and \\ divided into three $2.5 \mathrm{~min}$ blocks after pre-processing. Time effects for absolute and relative alpha activity \\ were found in healthy controls; these effects did not interact with AD/HD status. Interactions between \\ time and $A D / H D$ status were found for absolute theta, relative theta, and theta/beta ratio (TBR), with \\ these EEG indices increasing over time in children with AD/HD. Moreover, IQ played a role in the \\ interaction between time and $A D / H D$ status. These results are consistent with predictions from both the \\ optimal stimulation model and the delay aversion model, and suggest important methodological \\ considerations for future EEG research in children with/without AD/HD.

\section{Disciplines} \\ Education | Social and Behavioral Sciences

\section{Publication Details} \\ Zhang, D., Johnstone, S. J., Li, H., Barry, R. J., Clarke, A. R., Zhao, Q., Song, Y., Liu, L., Qian, Q., Wang, Y. \& \\ Sun, L. (2019). Time Effects on Resting EEG in Children With/Without AD/HD. Brain Topography: journal of \\ functional neurophysiology, 32 (2), 286-294.

\section{Authors} \\ Dawei Zhang, Stuart J. Johnstone, Hui Li, Robert J. Barry, Adam R. Clarke, Qihua Zhao, Yan Song, Lu Liu, \\ Qiujin Qian, Yufeng Wang, and Li Sun
}




\section{Time effects on resting EEG in children with/without AD/HD}

Da-Wei Zhang ${ }^{1,2}$, Stuart J. Johnstone ${ }^{1,2 *}$, Hui Li ${ }^{3,4}$, Robert J. Barry ${ }^{1,2}$, Adam R. Clarke ${ }^{1,2}$,

Qihua Zhao ${ }^{3,4}$, Yan Song ${ }^{5}$, Lu Liü,4, Qiujin Qian ${ }^{3,4}$, Yufeng Wang ${ }^{3,4}$, Li Sun ${ }^{3,4}$

${ }^{1}$ School of Psychology, University of Wollongong, Wollongong, NSW, Australia

${ }^{2}$ Brain \& Behaviour Research Institute, University of Wollongong, Wollongong, NSW,

Australia

${ }^{3}$ Peking University Sixth Hospital / Institute of Mental Health, Beijing, China

${ }^{4}$ National Clinical Research Center for Mental Disorders, Key Laboratory of Mental

Health, Ministry of Health, Peking University, Beijing, China

${ }^{5}$ State Key Laboratory of Cognitive Neuroscience and Learning \& IDG/McGovern

Institute for Brain Research, Beijing Normal University, China

\section{" Correspondence should be addressed to}

Stuart J. Johnstone

School of Psychology, University of Wollongong,

Wollongong, NSW 2522, Australia

e-mail:sjohnsto@uow.edu.au

Li Sun

Peking University Sixth Hospital

Beijing 100191, China

Email: sunlioh@bjmu.edu.cn 


\section{Acknowledgments}

This work was supported by the National Natural Sciences Foundation of China (81771479), the National Basic Research Program of China (973 program 2014CB846104), the Capital Characteristic Clinic Project (Z17110001017089). The Chinese Scholarship Council provided a scholarship for DW. Z. 


\begin{abstract}
In this study we extend on behavioural evidence to examine the effect of time on EEG measures related to arousal and emotion/motivation in children with/without AD/HD. Thirty children with AD/HD and 30 age- and sex-matched controls participated. EEG was recorded during an eyes-closed resting condition and divided into three 2.5 minute blocks after pre-processing. Time effects for absolute and relative alpha activity were found in healthy controls; these effects did not interact with AD/HD status. Interactions between time and $\mathrm{AD} / \mathrm{HD}$ status were found for absolute theta, relative theta, and theta/beta ratio (TBR), with these EEG indices increasing over time in children with $\mathrm{AD} / \mathrm{HD}$. Moreover, IQ played a role in the interaction between time and AD/HD status. These results are consistent with predictions from both the optimal stimulation model and the delay aversion model, and suggest important methodological considerations for future EEG research in children with/without AD/HD.
\end{abstract}




\section{Introduction}

Attention-deficit/Hyperactivity Disorder (AD/HD) is a common neurodevelopmental disorder characterized by inattention, hyperactivity, and impulsivity (APA 2000). The disorder often begins in early childhood and two-thirds of patients still show AD/HD symptoms in adulthood (Karam et al. 2015). The electroencephalogram (EEG) has been used to quantify central nervous system (CNS) activity in the disorder for several decades (Barry et al. 2003). Previous EEG studies reveal that patients with AD/HD typically show more low frequency activity and less high frequency activity than matched healthy controls during a resting state condition (Barry and Clarke 2009; Lenartowicz and Loo 2014); and these findings have been conceptualised as representing hypo-arousal, a maturational lag, or a developmental deviation (Barry et al. 2003; Saad et al. 2015). However, debate continues about whether $\mathrm{AD} / \mathrm{HD}$ can be reliably detected by EEG, as inconsistent results have been reported; e.g. AD/HD-control differences in the theta/beta ratio (TBR, Arns et al. 2013). Thus, EEG has not been regarded as a biological marker for the diagnosis of AD/HD (APA 2013).

Some factors are suggested to contribute to the inconsistent EEG results. A significant feature of EEG in AD/HD is that the patients show heterogeneous EEG profiles (Clarke et al. 2011; Loo et al. 2017). With this inter-individual variability, EEG comparison at the group level may be confounded. Moreover, patients with AD/HD often have comorbid issues, and these variations influence AD/HD vs. control EEG comparisons (Clarke et al. 2002; Loo et al. 2013). Further, it has been suggested that habit changes in controls (e.g. sleep duration) may have resulted in changes from AD/HD-control EEG differences reported in early studies (Arns et al. 2013).

More recently, EEG recording parameters have been considered as contributing to the inconsistent findings. Kitsune et al. (2015) showed that the EEG recording context in relation 
to other experimental demands influenced AD/HD-control EEG differences. This study suggests that the differences in EEG recording parameters between studies may contribute to the inconsistent findings and encourages further exploration. One such parameter is the duration of the EEG recording. Research shows that a minimum of 60 -seconds of artefactfree data can reliably quantify brain electrical activity for spectral power analysis (Thatcher 2010). In practice, typical recording lengths range from 2 to 5 minutes, and results from various recording durations are treated equally when compared between studies. An important underlying assumption is that brain state is stable across the recording durations in typically developing and clinical children.

However, behavioural findings from children in waiting situations indicate that this assumption - i.e. that brain activity is stable across time - may be violated. During a resting EEG recording, the participant has no specific task and is required to keep stationary and remain alert in a quiet, stimulation-free environment. This context is similar to a waiting task with no specific instructions or requirements in behavioural studies (e.g. Antrop et al. 2000, 2002). The behavioural research indicates that when children are asked to wait for a period in a low-stimulus situation, they show overactive and inattentive behaviours (Zentall and Zentall 1983; Antrop et al. 2000; Antrop et al. 2005) which increases frequently over time (Alberts and Van der Meere 1992; Imeraj et al. 2016). Moreover, AD/HD status exacerbates these behaviours, with evidence from both laboratory situations (Antrop et al. 2000, 2002) and naturalistic observations (Antrop et al. 2005; Imeraj et al. 2016). Taken together, the behavioural research suggests that children show inappropriate behaviours in waiting situations, and the effect is moderated by time and $\mathrm{AD} / \mathrm{HD}$ status.

The behaviours in waiting situations are suggested to have their roots in atypical CNS activity. The optimal stimulation model attributes the overactive and inattentive behaviours 
in waiting situations to arousal level (Zentall 1975; Zentall and Zentall 1983). According to this model, as sensory input and stimulation are reduced in waiting situations, CNS arousal level decreases over time. Consequently, children perform excessive behaviours as a “compensation” for the decreasing arousal level (Zentall and Zentall 1983; Antrop et al. 2000). Children with AD/HD, who commonly show low CNS arousal, are more sensitive to the low stimulation level, and as a result, more overactive and inattentive behaviour is seen (Zentall and Zentall 1983). In addition, for waiting situations in which participants are required to stay still over a period, the model predicts that arousal will more rapidly decrease as no "compensation” is received over time; which also interacts with AD/HD status (Zentall and Zentall 1983).

An alternative explanation for the exacerbation of $\mathrm{AD} / \mathrm{HD}$ symptoms in waiting situations is given in terms of motivation, via the delay aversion model (Sonuga-Barke 2003, 2005). Based on behavioural and imaging studies, the model hypothesizes in children with AD/HD a tendency to avoid delays as a result of impaired long-term reward systems. The delay aversion in turn drives children with AD/HD to avoid or escape the situations in which there is no instant reward. The attempts to avoid or escape are behaviourally manifested as overactivity and inattention (Sonuga-Barke 2005). Following this perspective, the overactivity and inattention of children with $\mathrm{AD} / \mathrm{HD}$ in waiting situations are attributed to motivational/emotional factors. Moreover, for a waiting situation that restricts attempts to avoid and escape (e.g. keeping stationary) over time, the model predicts children with AD/HD will show an increasingly stronger motivational/emotional reaction.

In the context of a resting EEG recording, participants are further instructed to stay as stationary as possible to reduce the influence of artifacts on the EEG traces. In other words, the resting EEG recording context could be described as a waiting situation in which “compensation” and the attempts to avoid are further prohibited. According to the optimal 
stimulation model and delay aversion model, brain activity related to arousal and motivation may be altered in children over time, with this effect moderated by AD/HD status.

As a tool to measure CNS activity, some EEG indices have been related to arousal level and motivational/emotional activity. Skin conductance level (SCL) is considered as a gold standard measure of arousal level (Barry and Sokolov 1993). Research has indicated that SCL is negatively correlated with absolute alpha EEG activity (Barry et al. 2004). The result has been replicated in different age ranges (Barry et al. 2007; Barry et al. 2009a) and in a stimulant-modulation study (Barry et al. 2005). Consequently, absolute alpha is regarded as an index to measure CNS arousal level (Barry et al. 2004). For motivation/emotion, candidate measures in EEG are the slow waves such as delta and theta (Knyazev 2007), particularly from reports that theta and delta activity vary in response to motivation/emotion related tasks (Knyazev et al. 2009; Womelsdorf et al. 2010).

Combining the above-mentioned behavioural findings and their CNS explanations, it is expected that EEG measures of arousal in children will vary over time, and this effect will interact with $\mathrm{AD} / \mathrm{HD}$ status. Also, EEG measures related to motivation/emotion will be altered in children with AD/HD over time. More specifically, it is predicted that: a) there will be an increase in alpha activity across time in children; b) the increase will be larger in children with AD/HD compared to controls; and c) there will be an increase in EEG slow waves across time in children with $\mathrm{AD} / \mathrm{HD}$.

\section{Materials and Methods}

\subsection{Participants}

Participants in the AD/HD group were selected from a database from the Institute of Mental Health at Peking University Sixth Hospital. The database included 102 cases and all cases were assessed and diagnosed with $\mathrm{AD} / \mathrm{HD}$ by experienced senior psychiatrists based on 
clinical observation and the results of the Clinical Diagnostic Interview Scale (CDIS, Barkley 1998). The CDIS is a structured clinical interview based on the DSM-IV. The mandarin version of CDIS, which shows good sensitivity and specificity (Yang et al. 2004), was administered by a psychiatrist with the participants and their parents. With the following inclusion criteria applied, thirty cases were selected from the database (25 males, age range 914 years, $M=11.5$ years, $S D=1.7$ ). The inclusion criteria included: 1 ) no comorbidity or history of psychiatric disorders described in the DSM-IV; 2) no history of head trauma with loss of consciousness; 3) no history of neurological illness or other severe disease; 4) naïve to any pharmacological treatment (the recordings were conducted when they were just diagnosed); 5) age ranging from 9 to 14; and 6) an IQ higher than 80 on the Wechsler Intelligence Scale III for children. Twenty one children with AD/HD were diagnosed with the predominantly inattentive type (ADHD-I) and 9 with the combined type (ADHD-C).

Thirty healthy controls were recruited from the hospital and local schools and were screened by the same psychiatrists using the same inclusion criteria. They were matched by age and gender (25 males, age range $9-14$ years, $M=11.9$ years, $S D=1.5$ ). Controls did not meet the diagnostic criteria for AD/HD (i.e. they met less than 4 DSM-IV Inattention criteria and less than 4 DSM-IV Hyperactivity/Impulsivity criteria).

\subsection{Procedure}

Ethical approval was obtained from the Ethics Committee of Peking University Health Science Center and the University of Wollongong Human Research Ethics Committee. Informed consent was obtained from the parent or guardian of each participant prior to accessing any record or testing.

Data were collected for this study on a separate day after the assessment and diagnostic session, with children required to complete an IQ test (Wechsler Intelligence Scale 
III) and neuropsychological tests accompanied by a psychiatrist in the morning, and resting EEG was recorded in the afternoon. This process lasted about 45 mins.

\subsection{EEG recording and pre-processing}

The EEG was recorded in a room with dimmed lighting which was free from distraction. Participants were seated in a comfortable chair and were required to keep their eyes closed; the recording was paused if the participant showed restlessness. EEG was recorded for between 7.5 and 10 minutes. The EEG was acquired using a 128-channel system (HydroCel Geodesic Sensor Net, Electrical Geodesics, Inc., Eugene, OR). The impedance of all electrodes was less than $50 \mathrm{k} \Omega$. All electrodes were physically referenced to Cz (fixed by the EGI system) and offline re-referenced to linked mastoids. The EEG was amplified with a band pass filter of 0.01 to $200 \mathrm{~Hz}$, and digitized on-line at a sampling rate of $1000 \mathrm{~Hz}$. The EGI data were converted to allow analysis using EEGLAB and Neuroscan software version 4.3.

To follow previous research (e.g. Barry and Clarke 2009), nineteen channels were selected based on the international 10-20 system and pre-processing steps were undertaken. All channels were re-sampled at $256 \mathrm{~Hz}$, and band-pass filtered from 1 to $70 \mathrm{~Hz}$ with a $50 \mathrm{~Hz}$ notch filter. Periods of the EEG trace that were affected by gross artefacts were identified by visual inspection and were excluded. The data then were subjected to an Independent Component Analysis in EEGLAB (Delorme and Makeig 2004) to identify and exclude components related to eye and muscle movements; this is a semi-automatic process aided by the ADJUST tool box in EEGLAB (Mognon et al. 2011).

Seven and a half minutes of artefact-free data were then extracted. Given the research purpose, EEG traces were chronologically divided into 3 blocks. Each block contained 2.5 minutes of EEG data and was segmented into 4-second epochs. The epochs then were Fourier transformed using a Hamming window. These epochs were Fourier transformed using a 
Hamming window, with EEG power obtained in four frequency bands: delta (1.5-3.5 Hz), theta $(3.5-7.5 \mathrm{~Hz})$, alpha $(7.5-12.5 \mathrm{~Hz})$, and beta $(12.5-25 \mathrm{~Hz})$. Absolute power was calculated by summing the spectral power within each frequency band, and relative power was calculated by dividing absolute power in each frequency band by the total of the four bands. Also, the theta/beta ratio (TBR) was calculated as it is a measure of continued interest in $\mathrm{AD} / \mathrm{HD}$ research.

\subsection{Statistical analysis}

The EEG data were subjected to mixed-design analyses of variance (ANOVAs) with Group (AD/HD, control) as a between-subjects factor and Time and Sagittal as withinsubjects factors. The analyses were separately conducted for the each frequency band for both absolute/relative power and TBR. The Time factor included 3 equal-length blocks (block 1 - T1, block 2 - T2, block 3 - T3). To follow previous studies (e.g. Clarke et al., 2011) the Sagittal factor consisted of frontal (Fp1, Fp2, F3, F4, F7, F8, Fz), central (C3, C4, $\mathrm{Cz}, \mathrm{T} 3, \mathrm{~T} 4)$, and posterior (T5, T6, P3, P4, Pz, O1, O2) regions.

Planned contrasts were examined for the within-subjects factors. To describe trends within the Time factor, planned polynomial contrasts were used. The planned contrasts were also used to examine EEG topography following previous studies (e.g. Clarke et al., 2006), comparing frontal (F) and posterior regions (P), and central region (C) with the mean of the frontal and posterior regions (F/P). As the contrasts are planned, and there are no more of them than the degrees of freedom for the effect, no Bonferroni-type adjustment to $\alpha$ is required (Tabachnick and Fidell 2007). Sagittal effects are reported only for interactions with Group or Group x Time interactions.

\section{Results}


Demographic information for the AD/HD and control groups is listed in Table 1. There was no significant age difference between groups. The control group had a higher IQ than the AD/HD group $(F=46.112, p<0.001)$. Although previous research showed that IQ level did not contribute to AD/HD-control EEG differences (Clarke et al., 2006), a recent study suggests that there may be an interaction between IQ and recording contexts on the differences (Kitsune et al. 2015). Hence, analyses involving the Group factor were run first with IQ uncontrolled, in line with many previous studies (e.g. Barry et al. 2009b), and then the analyses were conducted with IQ as a covariate.

The topographic maps for absolute power, relative power, and TBR for each Group over time are displayed in Fig. 1.

\section{- INSERT Table 1 HERE -}

\section{- INSERT Fig. 1. HERE -}

\subsection{Time effects}

No Time main effects were found. Near significant interactions between Time and Sagittal topography were found for absolute alpha power $(\mathrm{T} 3>\mathrm{T} 1 \times \mathrm{P}>\mathrm{F}, \mathrm{F}=3.200, p=$ $\left.0.079, \eta_{p}^{2}=0.052\right)$ and relative alpha power $\left(\mathrm{T} 3>\mathrm{T} 1 \times \mathrm{P}>\mathrm{F}, F=3.832, p=0.055, \eta_{p}^{2}=\right.$ 0.061). A close inspection of the data indicated that alpha activity had a larger standard deviation in the $\mathrm{AD} / \mathrm{HD}$ group, which may compromise the main effects. Hence, to characterise the Time effects in typically-developing children without the influence of AD/HD status, the analysis was re-conducted in only healthy controls. A Time $\times$ Sagittal interaction was present for absolute alpha power (T3 $>\mathrm{T} 1 \times \mathrm{P}>\mathrm{F}, F=5.290, p=0.029$, $\eta_{p}^{2}=0.154$ ) indicating a linear increase of absolute alpha in the posterior region (Fig. 2). A similar interaction was present for relative alpha power $(\mathrm{T} 3>\mathrm{T} 1 \times \mathrm{P}>\mathrm{F}, F=6.940, p=$ 0.013, $\left.\eta_{p}^{2}=0.193\right)$. No effects were present for the other bands or TBR.

\subsection{Group effects}


Compared to controls, children with AD/HD had more absolute delta $(F=4.992, p=$ 0.029, $\left.\eta_{p}^{2}=0.079\right)$, more relative delta $\left(F=7.426, p=0.008, \eta_{p}^{2}=0.105\right)$, and less relative alpha $\left(F=4.623, p=0.036, \eta_{p}^{2}=0.074\right)$. No interactions with the Sagittal factor were found, indicating that these effects were global. No effects were present for the other bands or TBR.

\subsection{Group $\times$ Time interaction effects}

A Group $\times$ Time interaction $\left(\mathrm{AD} / \mathrm{HD}>\right.$ control $\times \mathrm{T} 3>\mathrm{T} 1, F=4.416, p=0.040, \eta_{p}^{2}=$ 0.071) indicated that absolute theta was similar at T3 and T1 for controls but was larger at T3 than $\mathrm{T} 1$ in the $\mathrm{AD} / \mathrm{HD}$ group (Fig. 3). A similar interaction was present for relative theta $\left(\mathrm{AD} / \mathrm{HD}>\mathrm{control} \times \mathrm{T} 3>\mathrm{T} 1, F=6.161, p=0.016, \eta_{p}^{2}=0.096\right)$ and indicated that relative theta was similar T3 and T1 for controls but was larger at T3 than T1 in the AD/HD group (Fig. 3). The Group $\times$ Time interaction approached significance for TBR $(\mathrm{AD} / \mathrm{HD}>$ control $\left.\times \mathrm{T} 3>\mathrm{T} 1, F=3.615, p=0.062, \eta_{p}^{2}=0.059\right)$ and indicated that TBR was similar at T3 and T1 for controls but was larger at T3 than T1 in the AD/HD group (Fig. 3). No topographic effect was found for these interactions. No interactions were present for the other bands.

Although age and gender were matched between the groups, we double-checked the effects reported with age as a covariate and when excluding female participants, and the main results were unchanged.

\section{- INSERT Fig. 2. HERE -}

\section{- INSERT Fig. 3. HERE -}

\subsection{Group main effects with IQ controlled}

With IQ as a covariate, children with AD/HD still showed more absolute delta with marginal significance $\left(F=3.948, p=0.052, \eta_{p}^{2}=0.065\right)$, more relative delta $(F=8.528, p=$ 0.005, $\left.\eta_{p}^{2}=0.130\right)$, and less relative alpha $\left(F=4.159, p=0.046, \eta_{p}^{2}=0.068\right)$. 
With IQ as a covariate, the Group $\times$ Time interactions for absolute theta $(F=3.444, p$ $\left.=0.069, \eta_{p}^{2}=0.057\right)$ and relative theta $\left(F=3.681, p=0.060, \eta_{p}^{2}=0.061\right)$ only approached significance. The interaction for TBR no longer approached significance $(F=0.547, p=$ $\left.0.463, \eta_{p}^{2}=0.010\right)$

\section{Discussion}

The current study aimed to explore time effects in EEG spectral power recorded during resting conditions. EEG data were divided into 3 equal-length blocks. In light of behavioural findings under waiting situations, the current study predicted a time effect on alpha activity in children, which is moderated by $\mathrm{AD} / \mathrm{HD}$ status. Also, a Time $\mathrm{x} \mathrm{AD} / \mathrm{HD}$ status interaction was expected for low frequency activity.

The current study showed the effects of time on absolute and relative alpha in typically developed children. An increase of alpha activity was observed in this study. Previous research reported that alpha activity is negatively correlated with CNS arousal level. The inverse relationship was initially found in a study reporting that individuals with higher arousal level have a decreased alpha level (Barry et al. 2004). The result was subsequently replicated by findings that absolute alpha is negatively correlated with SCL in different ages (Barry et al. 2007, 2009a), and also by a study indicating that absolute alpha is suppressed with the intake of caffeine (Barry et al. 2005). Hence, the alpha activity increase reported here suggests that arousal level decreased over time in children.

The alpha increase is consistent with the predictions of the optimal stimulation model. The model hypothesizes that individuals tend towards an optimal arousal level - which is the peak zone in an inverse U-shape pattern relating arousal to comfort. Also, the model describes the relationship between stimulus input and CNS arousal level - CNS arousal level increases with greater stimulus input and vice versa. Stimulation modulation is the means to 
approach the optimal zone (Zentall 1975; Zentall and Zentall 1983). With low sensory stimulus input, stimulus-seeking behaviours (e.g. overactivity and inattention) are performed as a compensatory process to increase arousal level; without the compensatory behaviours, arousal level continues to decline. In order to obtain relatively artifact-free data, participants are usually required to not move while sitting in a quiet EEG recording room alone. In other words, the recording contexts are similar to waiting situations in which activity is further forbidden. As predicted by the optimal stimulation model, the current study shows the measure related to arousal decreases in such a situation.

Alpha varying as a function of time suggests that recording length should be considered when explaining alpha activity derived from a resting recording in children. To our knowledge, this is the first study to show EEG time effects in an arousal related measure recorded in children during resting conditions. The arousal decrease over time is in line with a preliminary analysis revealing a time effect on arousal measured by SCL (Barry et al. 2007). Resting alpha activity is widely used in research with children, often being regarded as a baseline. To obtain alpha activity immune to time effects, a minimum recording length that is long enough for quantifying data is recommended. In cases where a lengthy recording is necessary, for example using both eyes-closed condition and eyes-open condition as baseline (Barry et al. 2009), a balanced design is suggested rather than a fixed order (e.g. eyes-closed data first, followed by eyes-open recording) to control the time effect. Moreover, the context of resting EEG recording in fact represents a situation with low sensory input. The results may also be applied for EEG recorded during tasks in which participants are instructed to continuously process repetitive and simple stimuli.

Inconsistent with our prediction, however, the time effect on alpha activity did not interact with $\mathrm{AD} / \mathrm{HD}$ status. According to the optimal stimulation model, children with AD/HD are more sensitive to situations with low stimulus input (Zentall 1975; Zentall and 
Zentall 1983), thus a greater decrease in arousal level would be expected over time. Our finding may be the result of compensation by the $\mathrm{AD} / \mathrm{HD}$ group in response to low stimulus input not being well controlled. According to the optimal stimulus model, inattention and overactivity react as compensation to a low level arousal state for children with AD/HD. In our study, participants were required to keep as still as possible and only obvious movements were monitored. However, research using actigraphy devices has reported that children with $\mathrm{AD} / \mathrm{HD}$ have excessive fine movements, such as wrist- and ankle-related movements (Wood et al. 2009; Alderson et al. 2012; Gilbert et al. 2016). These movements were not controlled here and may have been used as compensation to increase arousal in the $\mathrm{AD} / \mathrm{HD}$ group. In addition to physical activity, children with AD/HD may not be not mentally rest, which can also impact on arousal.

An alternate explanation is the heterogeneous nature of AD/HD. Prevailing models attribute the deficits of AD/HD to different sources (Sonuga-Barke 2005; Sergeant 2005; Castellanos et al. 2006). Following this perspective, it is possible that not all children with AD/HD have a decreased arousal level, which is consistent with EEG findings that children with AD/HD show different profiles of alpha activity (Barry et al. 2003; Clarke et al. 2011). Indeed, larger variance in alpha activity across time was shown in the AD/HD group. Hence, the hypothesis may be possible only in children with AD/HD having the deficit of hypo-arousal.

There was an interaction between time and $\mathrm{AD} / \mathrm{HD}$ status for theta activity compared to healthy controls, children with AD/HD showed an increasing trend of relative and absolute theta over time. As beta activity remained similar across time, the marginal significance for TBR increasing over time is likely driven by the theta increase. The theta finding is in line with the prediction derived from the delay aversion model, as theta activity is associated with motivational and emotional activity. A substantial number of studies report 
that theta activity is increased in the presence of emotional stimuli (Knyazev 2007; Knyazev et al. 2009; Uusberg et al. 2014) and in response to motivational activity (Knyazev 2007) such as during approach behaviours (Walden et al. 2015). From this perspective, the increased theta activity reported here may reflect the increasingly activated motivational/emotional cortical activity in children with $\mathrm{AD} / \mathrm{HD}$ over time, which is consistent with the prediction of the delay aversion model. The model predicts that children with $\mathrm{AD} / \mathrm{HD}$ have impaired long-term rewarding system and consequently avoid staying in situations without instant rewards (Sonuga-Barke 2005). Hence, the long-period EEG recording in this study may act as a trigger that activates motivational/emotional response in children with AD/HD.

Consistent with a previous study (Clarke et al. 2006), this study showed that the Group effect was not influenced by IQ. However, with IQ controlled the previous significant Time x Group interactions for relative and absolute theta remained but reduced to a trend level, and a substantial influence was shown for TBR with the previously significant interaction trend disappearing. These changes indicate that IQ interacted with the Time and AD/HD status had an influence on theta activity and TBR. TBR has been suggested as a biomarker to differentiate AD/HD (Snyder and Hall 2006); however, inconsistent results are reported (Arns et al. 2013). As children with AD/HD typically show lower IQ, this study suggests that IQ should be controlled when long period EEG data are analysed.

Some of the issues emerging from the theta and TBR findings relate to differentiating children with AD/HD from controls. EEG offers hope of objectively diagnosing AD/HD (Barry and Clarke 2009); however, inconsistent results have been reported, for example in theta and TBR (Arns et al. 2013; Barry and Clarke 2009; Lenartowicz and Loo 2014). The current findings suggest that recording length together with IQ affects theta activity and consequently TBR. Hence, the role of recording length interacting with IQ could be 
considered to reconcile the inconsistent results, as these factors were not strictly controlled in previous research. Meanwhile, this study also contributes to research considering the optimal recording situation to reveal $\mathrm{AD} / \mathrm{HD}$-control differences - raised by a recent study (Kitsune et al. 2015). This current study suggests that the group differences for theta and TBR tend to enlarge when the groups are exposed to a long resting EEG recording. In this case, the duration of the resting EEG recording is added as a factor to elicit the difference between the groups.

The generalisability of this study is subject to certain limitations. The 3 blocks were divided after raw EEG data had been screened to exclude artefacts-related EEG traces. Hence, the 3 blocks do not necessarily exactly represent the first 2.5-minutes period, the second 2.5-minutes period, and the third 2.5-minutes period. In addition, although participants were instructed not to move and their activity was monitored, an additional uncontrolled aspect is that it is unknown whether children with AD/HD performed more fine motor activities, which may be a compensation for the decreasing arousal as introduced above. Moreover, theta activity in this study was explained based by the framework of its relationships with motivation/emotion (Knyazev 2007). However, theta activity may also reflect other internal processing such as working memory (Sauseng et al. 2010), which leads to the possibility that the theta increase is driven by processing other than motivation/emotion. Thus, studies may further explore the mechanism of the theta increase.

In conclusion, with the similarity between EEG recording contexts and waiting situations, this study examined time effects on EEG measures in children with/without AD/HD. An effect of time on alpha activity was observed in children. In addition, the effect of time on theta activity and TBR was moderated by AD/HD status. Moreover, IQ played a role in the interaction between time and $\mathrm{AD} / \mathrm{HD}$ status. Overall, these findings have methodological implications for EEG research in children with/without AD/HD. 


\section{References}

Alberts, E., \& van der Meere, J. (1992). Observations of hyperactive behaviour during vigilance. J Child Psychol Psychiatry, 33(8), 1355-1364.

Alderson, R. M., Rapport, M. D., Kasper, L. J., Sarver, D. E., \& Kofler, M. J. (2012). Hyperactivity in boys with attention deficit/hyperactivity disorder (ADHD): the association between deficient behavioral inhibition, attentional processes, and objectively measured activity. Child Neuropsychol, 18(5), 487-505.

American Psychiatric Association., \& American Psychiatric Association. DSM-5 Task Force. (2013). Diagnostic and statistical manual of mental disorders : DSM-5 (5th ed.). Washington, D.C.: American Psychiatric Publishing.

American Psychiatric Association., \& American Psychiatric Association. Task Force on DSM-IV. (2000). Diagnostic and statistical manual of mental disorders : DSM-IV-TR (4th ed.). Washington, DC: American Psychiatric Association.

Antrop, I., Buysse, A., Roeyers, H., \& Van Oost, P. (2002). Stimulation seeking and hyperactive behavior in children with ADHD: a re-analysis. Percept Mot Skills, 95(1), 71-90. doi:10.2466/pms.2002.95.1.71

Antrop, I., Buysse, A., Roeyers, H., \& Van Oost, P. (2005). Activity in children with ADHD during waiting situations in the classroom: a pilot study. Br J Educ Psychol, 75(Pt 1), 51-69.

Antrop, I., Roeyers, H., Van Oost, P., \& Buysse, A. (2000). Stimulation seeking and hyperactivity in children with ADHD. Attention Deficit Hyperactivity Disorder. J Child Psychol Psychiatry, 41(2), 225-231.

Arns, M., Conners, C. K., \& Kraemer, H. C. (2013). A decade of EEG Theta/Beta Ratio Research in ADHD: a meta-analysis. J Atten Disord, 17(5), 374-383.

Barkley, R. A. (1998). Attention-deficit hyperactivity disorder : a handbook for diagnosis and treatment (2nd ed.). New York: Guilford Press.

Barry, R. J., Clarke, A. R., \& Johnstone, S. J. (2003). A review of electrophysiology in attention-deficit/hyperactivity disorder: I. Qualitative and quantitative electroencephalography. Clin Neurophysiol, 114(2), 171-183.

Barry, R. J., Clarke, A. R., Johnstone, S. J., \& Brown, C. R. (2009a). EEG differences in children between eyes-closed and eyes-open resting conditions. Clin Neurophysiol, 120(10), 1806-1811.

Barry, R. J., Clarke, A. R., Johnstone, S. J., Magee, C. A., \& Rushby, J. A. (2007). EEG differences between eyes-closed and eyes-open resting conditions. Clin Neurophysiol, 118(12), 2765-2773. 
Barry, R. J., Clarke, A. R., Johnstone, S. J., McCarthy, R., \& Selikowitz, M. (2009b). Electroencephalogram theta/beta ratio and arousal in attention-deficit/hyperactivity disorder: evidence of independent processes. Biol Psychiatry, 66(4), 398-401.

Barry, R. J., Clarke, A. R., McCarthy, R., Selikowitz, M., Rushby, J. A., \& Ploskova, E. (2004). EEG differences in children as a function of resting-state arousal level. Clin Neurophysiol, 115(2), 402-408.

Barry, R. J., Rushby, J. A., Wallace, M. J., Clarke, A. R., Johnstone, S. J., \& Zlojutro, I. (2005). Caffeine effects on resting-state arousal. Clin Neurophysiol, 116(11), 26932700 .

Barry, R. J., \& Sokolov, E. N. (1993). Habituation of phasic and tonic components of the orienting reflex. Int J Psychophysiol, 15(1), 39-42.

Castellanos, F. X., Sonuga-Barke, E. J., Milham, M. P., \& Tannock, R. (2006). Characterizing cognition in ADHD: beyond executive dysfunction. Trends Cogn Sci, 10(3), 117-123.

Clarke, A. R., Barry, R. J., Dupuy, F. E., Heckel, L. D., McCarthy, R., Selikowitz, M., \& Johnstone, S. J. (2011). Behavioural differences between EEG-defined subgroups of children with Attention-Deficit/Hyperactivity Disorder. Clin Neurophysiol, 122(7), 1333-1341.

Clarke, A. R., Barry, R. J., McCarthy, R., \& Selikowitz, M. (2002). Children with attentiondeficit/hyperactivity disorder and comorbid oppositional defiant disorder: an EEG analysis. Psychiatry Res, 111(2-3), 181-190.

Clarke, A. R., Barry, R. J., McCarthy, R., Selikowitz, M., Magee, C. A., Johnstone, S. J., \& Croft, R. J. (2006). Quantitative EEG in low-IQ children with attentiondeficit/hyperactivity disorder. Clin Neurophysiol, 117(8), 1708-1714.

Delorme, A., \& Makeig, S. (2004). EEGLAB: an open source toolbox for analysis of singletrial EEG dynamics including independent component analysis. J Neurosci Methods, 134(1), 9-21.

Gilbert, H., Qin, L., Li, D., Zhang, X., \& Johnstone, S. J. (2016). Aiding the diagnosis of AD/HD in childhood: Using actigraphy and a continuous performance test to objectively quantify symptoms. Res Dev Disabil, 59, 35-42.

Imeraj, L., Antrop, I., Roeyers, H., Deboutte, D., Deschepper, E., Bal, S., \& Sonuga-Barke, E. (2016). The Impact of Idle Time in the Classroom: Differential Effects on Children With ADHD. J Atten Disord, 20(1), 71-81.

Karam, R. G., Breda, V., Picon, F. A., Rovaris, D. L., Victor, M. M., Salgado, C. A., . . Bau, C. H. (2015). Persistence and remission of ADHD during adulthood: a 7-year clinical follow-up study. Psychol Med, 1-12.

Kitsune, G. L., Cheung, C. H., Brandeis, D., Banaschewski, T., Asherson, P., McLoughlin, G., \& Kuntsi, J. (2015). A Matter of Time: The Influence of Recording Context on 
EEG Spectral Power in Adolescents and Young Adults with ADHD. Brain Topogr, 28(4), 580-590.

Knyazev, G. G. (2007). Motivation, emotion, and their inhibitory control mirrored in brain oscillations. Neurosci Biobehav Rev, 31(3), 377-395.

Knyazev, G. G., Slobodskoj-Plusnin, J. Y., \& Bocharov, A. V. (2009). Event-related delta and theta synchronization during explicit and implicit emotion processing. Neuroscience, 164(4), 1588-1600.

Lenartowicz, A., \& Loo, S. K. (2014). Use of EEG to diagnose ADHD. Curr Psychiatry Rep, 16(11), 498.

Loo, S. K., Cho, A., Hale, T. S., McGough, J., McCracken, J., \& Smalley, S. L. (2013). Characterization of the theta to beta ratio in ADHD: identifying potential sources of heterogeneity. J Atten Disord, 17(5), 384-392.

Loo, S. K., McGough, J. J., McCracken, J. T., \& Smalley, S. L. (2017). Parsing heterogeneity in attention-deficit hyperactivity disorder using EEG-based subgroups. J Child Psychol Psychiatry.

Mognon, A., Jovicich, J., Bruzzone, L., \& Buiatti, M. (2011). ADJUST: An automatic EEG artifact detector based on the joint use of spatial and temporal features. Psychophysiology, 48(2), 229-240.

Sauseng, P., Griesmayr, B., Freunberger, R., \& Klimesch, W. (2010). Control mechanisms in working memory: A possible function of EEG theta oscillations. Neurosci Biobehav Rev, 34(7), 1015-1022.

Saad, J. F., Kohn, M. R., Clarke, S., Lagopoulos, J., \& Hermens, D. F. (2015). Is the Theta/Beta EEG Marker for ADHD Inherently Flawed? J Atten Disord.

Sergeant, J. A. (2005). Modeling attention-deficit/hyperactivity disorder: a critical appraisal of the cognitive-energetic model. Biol Psychiatry, 57(11), 1248-1255.

Snyder, S. M., \& Hall, J. R. (2006). A meta-analysis of quantitative EEG power associated with attention-deficit hyperactivity disorder. J Clin Neurophysiol, 23(5), 440-455.

Sonuga-Barke, E. J. (2003). The dual pathway model of AD/HD: an elaboration of neurodevelopmental characteristics. Neurosci Biobehav Rev, 27(7), 593-604.

Sonuga-Barke, E. J. (2005). Causal models of attention-deficit/hyperactivity disorder: from common simple deficits to multiple developmental pathways. Biol Psychiatry, 57(11), 1231-1238.

Tabachnick, B. G., \& Fidell, L. S. (2007). Using multivariate statistics (5th ed.). Boston: Pearson/Allyn \& Bacon.

Thatcher, R. W. (2010). Validity and Reliability of Quantitative Electroencephalography. Journal of Neurotherapy, 14, 122-152. 
Uusberg, A., Thiruchselvam, R., \& Gross, J. J. (2014). Using distraction to regulate emotion: insights from EEG theta dynamics. Int J Psychophysiol, 91(3), 254-260.

Walden, K., Pornpattananangkul, N., Curlee, A., McAdams, D. P., \& Nusslock, R. (2015). Posterior versus frontal theta activity indexes approach motivation during affective autobiographical memories. Cogn Affect Behav Neurosci, 15(1), 132-144.

Womelsdorf, T., Vinck, M., Leung, L. S., \& Everling, S. (2010). Selective thetasynchronization of choice-relevant information subserves goal-directed behavior. Front Hum Neurosci, 4, 210.

Wood, A. C., Asherson, P., Rijsdijk, F., \& Kuntsi, J. (2009). Is overactivity a core feature in ADHD? Familial and receiver operating characteristic curve analysis of mechanically assessed activity level. J Am Acad Child Adolesc Psychiatry, 48(10), 1023-1030.

Yang, L., Wang, Y. F., Qian, Q. J., Biederman, J., \& Faraone, S. V. (2004). DSM-IV subtypes of ADHD in a Chinese outpatient sample. J Am Acad Child Adolesc Psychiatry, 43(3), 248-250.

Zentall, S. (1975). Optimal stimulation as theoretical basis of hyperactivity. Am J Orthopsychiatry, 45(4), 549-563.

Zentall, S. S., \& Zentall, T. R. (1983). Optimal stimulation: a model of disordered activity and performance in normal and deviant children. Psychol Bull, 94(3), 446-471. 
Table 1 Demographic information for the groups. Numbers in brackets represent standard deviation.

\begin{tabular}{lllllll}
\hline & Gender & Age & IQ & $\begin{array}{l}\text { AD/HD } \\
\text { subtype }\end{array}$ & IN & HI \\
\hline AD/HD & $25 \mathrm{M}$; 5 F & $11.5(1.7)$ & $105.6(12.1)$ & 21 I; 9 C & $26.9(3.6)$ & $21.2(6.2)$ \\
Controls & $25 \mathrm{M}$; 5 F & $11.9(1.5)$ & $124.3(8.9)$ & - & - & - \\
\hline
\end{tabular}

Abbreviations: IN and HI, the inattention score and the hyperactivity-impulsivity score in AD/HD Rating Scale. 


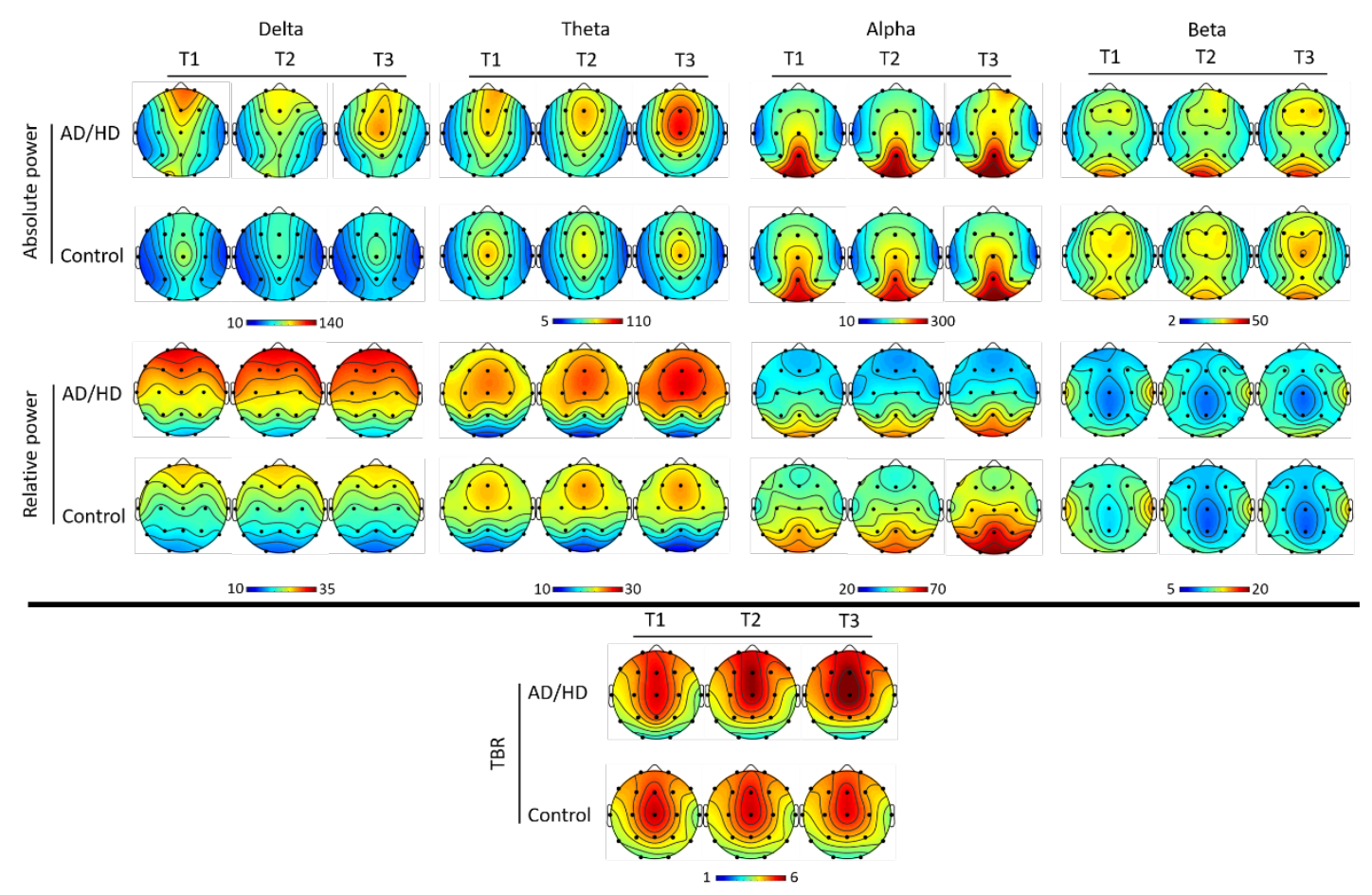

Fig. 1. The topographic maps for absolute power $\left(\mu \mathrm{V}^{2}\right)$, relative power (\%), and TBR for each group over time. 


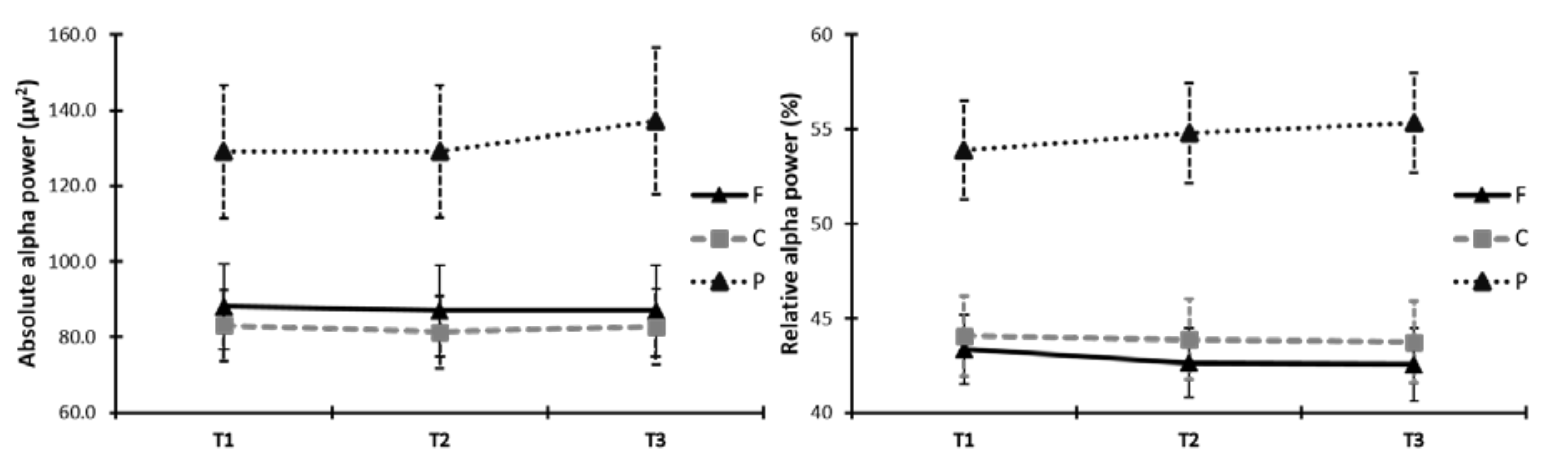

Fig. 2. The Time $\times$ Sagittal interaction of absolute alpha power (left panel) and relative alpha power (right panel) in the controls. 


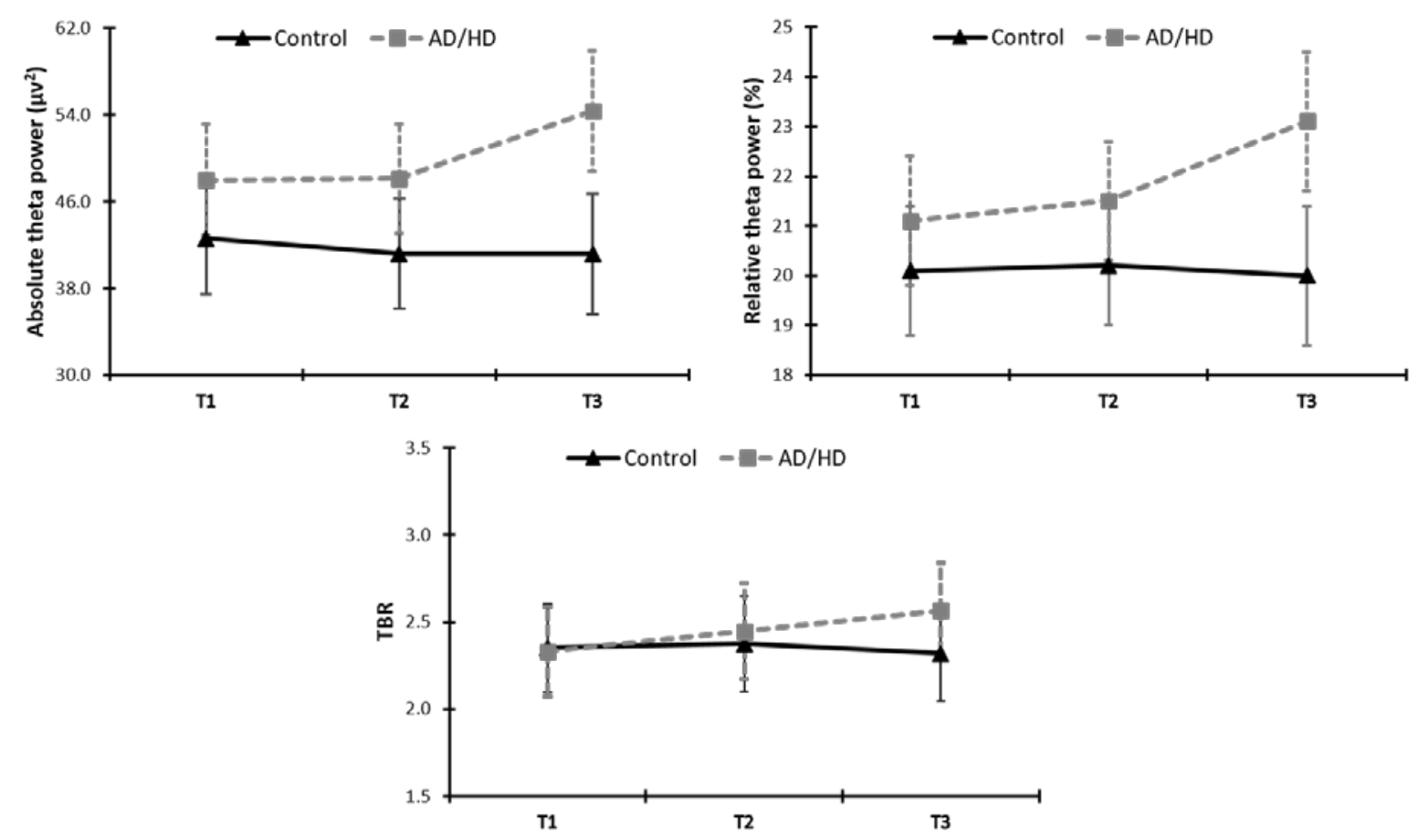

Fig. 3. The Group $\times$ Time interactions of absolute theta power (top left panel), relative theta (top right panel), and TBR (bottom panel) 